\title{
ANATOMY OF NERVES OF THE LOWER EXTREMITIES IN THE CONTEXT OF THE TREATMENT FOR VARICOSE VEINS
}

\author{
Dariusz Kowalczyk' ${ }^{1}$ Kamil Kowalczyk², Marian Simka' \\ 'Department of Anatomy, Institute of Medicine, University of Opole, Opole, Poland \\ Jan Mikulicz-Radecki University Teaching Hospital, Wrocław, Poland
}

\begin{abstract}
Injury of nerves of the lower extremity is the most prevalent complication associated with invasive treatment of varicose veins. These adverse events are particularly frequent after traditional surgical stripping and thermal ablative procedures. The most frequently injured nerves comprise the saphenous nerve, the sural nerve, and the anterior cutaneous branch of the femoral nerve. The high rate of injury to these nerves is primarily related to their close anatomical relationship with major trunks of the superficial venous system. The great saphenous vein is accompanied by two groups of sensory nerves. In its proximal part it runs along the anterior cutaneous branch of the femoral nerve. In some individuals, also the cutaneous branch of the obturator nerve is situated close to the proximal part of the great saphenous vein. The distal part of the great saphenous vein is accompanied by the saphenous nerve, a branch of the femoral nerve. The sural nerve, which typically consists of its medial and lateral branches, runs along the small saphenous vein. Also, during thermal ablation of the small saphenous vein the branches of the sciatic nerve containing motor fibres, the tibial and/or common peroneal nerves can also be injured. In this review the topographic anatomy of nerves accompanying the veins of the lower extremity is described. It is also discussed how to minimise the risk of these neurologic complications, knowing the topography, embryological development, and anatomical variability of veins and nerves in this part of the human body.
\end{abstract}

Key words: anatomy, anterior cutaneous branch of femoral nerve, nerve injury, saphenous nerve, sural nerve, surgery, varicose veins.

\author{
REVIEW PAPER \\ Phlebological Review 2019; 27, 1: 10-14 \\ DOl: https://doi.org/10.5114/pr.2019.93403
}

Submitted: 10.09 .2019

Accepted: 8.11.2019

\author{
ADDRESS FOR CORRESPONDENCE \\ Marian Simka \\ Department of Anatomy \\ Faculty of Medicine \\ University of Opole \\ 11 A Kopernika PI. \\ 45-040 Opole, Poland \\ e-mail: mariansimka@poczta.onet.pl
}

\section{INTRODUCTION}

Over the last two decades there was a substantial change in the treatment of varicose veins - a shift from traditional surgical excision to less invasive, primarily endovenous, techniques. Consequently, the longterm results of treatment improved, and the procedures became more acceptable to patients. Nonetheless, injuries to the nerves remain the most prevalent complications following treatment for varicose veins, both associated with surgical stripping [1-5] and with endovenous thermal ablations [6-10]. In this review we describe the anatomy of the nerves accompanying major veins of the lower extremity in the context of possible damage of these nerves during the treatment for varicose veins. We will also discuss how to minimise the risk of these complications, knowing the topography, embryological development, and anatomical variability of veins and nerves in this part of the human body.

\section{VARICOSE VEINS AND TOPOGRAPHICAL ANATOMY OF THE VEINS AND NERVES OF THE LOWER EXTREMITIES}

The majority of clinically relevant varicosities are associated with incompetence of either the great saphenous vein (GSV) or the small saphenous vein (SSV). These two veins belong to the superficial venous system of the lower limb but - unlike other veins of this system - are situated in the interfascial compartment (visible as an "Egyptian eye" in the transverse sonographic scan). This fascial compartment is separated by the superficially located saphenous fascia and the deep fascia covering adjacent muscles $[11,12]$. Other, similarly veins of the lower limb with interfascial location comprise the anterior accessory saphenous vein and the thigh extension of the small saphenous vein (referred to as the vein of Giacomini if this vein cranially joins the GSV). Nevertheless, invasive treatment of these veins is rarely associated 
with nerve injuries and therefore these veins will not be discussed in this paper.

The GSV is accompanied by two groups of sensory nerves. In its proximal part it runs along the anterior cutaneous branch of the femoral nerve (ACBFN). In some individuals also the cutaneous branch of the obturator nerve is situated close to the proximal part of the GVS. The distal part of the GSV is accompanied by the saphenous nerve, a branch of the femoral nerve $[13,14]$. Since all these nerves are located in the interfascial compartment and are quite close to the GSV, they can be quite easily injured during traditional surgery and endovenous thermal ablations of this vein.

\section{INJURIES OF THE ANTERIOR CUTANEOUS BRANCH OF THE FEMORAL NERVE}

Anatomical relationships between the ACBFN and the GSV are highly variable. This nerve typically accompanies the GSV in the proximal and middle part of the thigh, but the area of the saphenofemoral junction is the place where it is typically very close to the GSV and can be injured by the surgeon. Therefore, traditional stripping with high ligation of the GSV and closure of all tributaries of the GSV in the groin, which requires extensive surgical dissection of this area, is quite often associated with injury of the ACBFN. Such an injury clinically manifests with paraesthesia or dysaesthesia in the groin and/or middle aspect of the thigh. Although precise data are not available, endovenous techniques are probably associated with fewer injuries of the ACBFN because the most proximal part of the GSV (usually, $2 \mathrm{~cm}$ from the saphenofemoral junction), except for some non-standard laser techniques, is not obliterated during thermal ablation. Neurological complications are also possible in patients presenting with the ACBFN running close to the GVS in the middle part of the thigh. Also, in some individuals the GVS in the middle or distal part of the thigh is accompanied by the cutaneous branch of the obturator nerve [14], with possible injury of this nervous branch, which is anatomically and clinically similar to the injuries of the ACBFN. Anatomical variants characterised by nerves running close to the GSV in the middle part of the thigh seem to be particularly susceptible to neurological complications during invasive treatment of varicose veins in the GSV territory because doctors usually do not pay much attention to this part of the GSV during the procedure. Stripping of the mid-femoral part of the GSV is performed in a blinded manner, and, similarly to during thermal ablations, doctors do not look routinely for nerves adjacent to the GSV. Nevertheless, the use of tumescent anaesthesia during endovenous procedures probably minimises the risk of nerve injury in this area. The GSV is located within the interfascial compartment. Local tumescent anaesthesia injected into the interfascial compartment, next to the GSV, anaesthetises the nerves. In addition, anaesthetic fluid separates the vein from adja- cent anatomical structures, including the nerves, and also absorbs thermal energy. In this way it prevents unwanted damage of tissues located outside the target vein [15]. Nowadays, general or spinal anaesthesia is rarely used during the treatment for varicose veins, not only regarding endovenous procedures where local tumescent anaesthesia is the standard, but also during traditional stripping [16]. Consequently, paraesthesias in the groin area and middle aspect of the thigh are rarely reported in recent medical literature. Besides, dermatomes of particular sensory nerves of the thigh usually overlap, and therefore neurological deficits after injury of the above-mentioned nerves are not seen or are only transient.

\section{INJURIES OF THE SAPHENOUS NERVE}

Injury of the saphenous nerve is the most frequent neurological complication after the treatment for varicose veins. Injury of this nerve occurs in about $50 \%$ of patients after traditional stripping of the GSV with the use of old-fashioned strippers, also in approximately $30 \%$ of patients managed with thermal ablation devices or minimally-invasive stripping techniques. Nonetheless, some authors report much lower incidence of saphenous nerve injury after surgical treatment of varicose veins [1]. Importantly, injury of this nerve is probably highly under-reported because in the majority of patients the clinical symptoms are mild and/or transient [4, 5, 17, 18]. However, in some patients these symptoms are persistent and quite severe. Consequently, they are the main cause of litigation after vascular surgical treatments [3]. The incidence of such clinically relevant nerve injuries after surgical stripping is at the level of 3-9\%, after laser ablation - $0-3 \%$, and after RF closure of varicose veins - 4-6\% [19, 20]. In the proximal thigh the saphenous nerve cannot be damaged during the treatment because it runs along the distal part of the femoral artery. However, just above the knee, this nerve leaves the adductor canal, becomes a superficial structure and runs along the GSV. In the upper part of its superficial course it lies under the crural fascia, and then, usually in the upper third of the leg, it pierces the fascia and runs in the subcutaneous compartment. In the majority of people, the saphenous nerve becomes progressively closer to the GSV as it descends distally [14, 21]. Moreover, a close adhesion of this nerve to the GSV is more often seen if this nerve pierces the fascia more proximally than usually, just below the patella. Such a high location of the piercing of fascia is seen in about $25 \%$ of humans [14]. Injury of the saphenous nerve results in sensory disturbances in the medial malleolar region [20]. Because of a typical adhesive relationship between this nerve and the GSV, tumescent anaesthesia cannot adequately separate the saphenous nerve from the vein in the lower leg. Thus, there is a high incidence of neurological complications if surgical excisions or thermal ablations are performed below the knee $[20,22]$. Besides, the saphenous nerve, 
distally from the adductor canal, gives off the infrapatellar branch. The infrapatellar branch of the saphenous nerve is a sensory nerve, which innervates the anterior aspect of the knee, the anterolateral aspect of the lower leg in its proximal portion, and also part of the joint capsule of the knee. Injury of this branch results in paraesthesias in the medial aspect of the knee, which - in addition to the region of the medial malleolus - is a typical area affected by sensory disturbances after varicose vein surgery. This nervous branch not only can be injured during stripping of the GSV, but it is also at risk during stab avulsions of varicosities localised in the medial aspect of the knee. An injury of the infrapatellar branch can result in knee pain, and such adverse events have been reported after orthopaedic surgery procedures in this region [23]. Although knee pains after varicose vein surgery were not described in the medical literature, perhaps such a complication actually occurs in some patients but is improperly interpreted as being associated with knee degeneration (gonarthrosis is quite prevalent in varicose vein patients; therefore, such a misdiagnosis is likely).

\section{INJURIES OF THE SURAL NERVE}

Treatment of varicose veins in the SSV territory can be associated with injuries of the sural nerve. Prevalence of sural nerve injury after SSV stripping or thermal ablation is not precisely known. Probably it is at the level of around $20 \%[8,20]$ and is similar to the injuries of the saphenous verve. The majority of cases are mild and/ or temporary. Because of the anatomy of this nerve, in many patients this complication is difficult to avoid. The sural nerve, which typically consists of its medial and lateral branches, runs along the SSV [24]. Injury of this nerve can result in unpleasant sensations in the area of the lateral ankle and lateral aspect of the foot. Similarly to the saphenous nerve, the sural nerve is typically the closest to the SSV in the distal part of the lower leg [25]. Fortunately, an excision of varicose veins is rarely performed in the lateral ankle area, because usually only the proximal and middle parts of the SSV are pathologically dilated. If a distal part of incompetent SSV should also be ablated, it is recommended to visualise the sural nerve and separate it from the SSV with tumescent anaesthetic fluid under sonographic guidance [26]. Because of a high risk of sural nerve injury in this area, the distal part of the SSV should not be ablated if this part of the vein is not pathologically dilated or refluxing [7]. Typically, the most vulnerable is the middle part of the sural nerve, which is close to the SSV, although it rarely strictly adheres to the vein [9, 24, 26-29]. A lower incidence of sural nerve injuries in its proximal part is associated with the fact that usually in this area the nerve is not situated inside the interfascial compartment but is separated from the SSV by the muscular fascia. Conversely, in the middle part of the lower leg at least the medial branch of the sural nerve runs in the interfascial compartment, together with the SSV [24]. In comparison with the interfascial compartment containing the GSV in the thigh, the interfascial compartment containing the SSV in the lower leg is relatively small. Consequently, typically there is not enough space for adequate separation of this nerve from the SSV by anaesthetic fluid. This may explain the high incidence of injuries of the sural nerve after thermal ablation techniques. The same problem is seen during surgical stripping of the SSV - in most of the cases there is not enough place in the interfascial compartment to perform a gentle stripping. In the proximal part of the SSV the sural nerve usually does not strictly adhere to the vein. Yet, injuries of this vein at the saphenopopliteal junction are also likely, especially during open surgery. Traditional surgery recommends flush ligation of the SSV at the saphenopopliteal junction, similarly to the treatment of varicosities associated with GSV incompetence. This, however, requires an extensive dissection of the popliteal fossa, with high risk of injury not only of the sural nerve, but even of motor fibres of the sciatic nerve.

\section{INJURIES OF MOTOR BRANCHES OF THE SCIATIC NERVE}

Branches of the sciatic nerve containing motor fibres, the tibial, and/or common peroneal nerves can also be injured during thermal ablation of the SSV if such a procedure is performed inadequately. Although this complication is rare, neurological deficits are often permanent and severe [30-32]. Such an injury is possible, because the arch of the SSV - the most proximal part of this vein - is very close to the sciatic nerve (or its branches, depending on the level of division of the sciatic nerve). In addition, the proximal part of the sural nerve usually crosses the arch of the SSV and can be damaged during thermal ablation performed close to the saphenopopliteal junction or during flush ligation of the SSV [28]. Of note, topography of the nerves in the popliteal fossa, in relation to the saphenopopliteal junction and proximal part of the SSV, is highly variable. Thus, a risk of nerve injury during surgical dissection or thermal ablation next to the saphenopopliteal junction is high [33]. Moreover, an injection of aesthetic fluid may displace anatomical structures in the popliteal fossa. Even if prior to the injection of tumescent anaesthesia the vein was located far away from the nerves, after an administration of aesthetic fluid the nerves can be moved dangerously close to the SSV. Therefore, it is advisable to check anatomical relationships in the popliteal fossa again after completion of tumescent anaesthesia [34].

\section{HOW TO AVOID NERVE INJURIES DURING THE TREATMENT OF VARICOSE VEINS}

In the past there was a discussion as to whether the direction of stripping (upwards or downwards) is asso- 
ciated with different risk of nerve injuries. It was recommended that downward instead of an upward stripping be performed. Actually, there is no sufficient evidence supporting any direction of stripping $[35,36]$. On the other hand, low-invasive stripping, such as invagination technique or the use of pin-strippers, may reduce the rate of these neurological complications [2]. Because nowadays surgical techniques are replaced by endovenous procedures, this discussion becomes obsolete.

Although, in comparison with traditional surgery, there seem to be fewer neurological adverse events after endovenous techniques, the frequency of these complications is still unacceptably high [19]. One may ask why there are so many nerve injuries after invasive treatment for varicose veins. Because of embryologic development, major veins of the lower extremity run in the proximity of nerves (the so-called angiogenic nerves) [28]. In some areas the veins and nerves are situated particularly close to each other. The most vulnerable nerves that can be injured during the treatment for varicose veins have been described in the previous chapter of this paper. Murakami et al. [14] have histologically studied nerves that were macroscopically located close to the GSV of SSV. They found that nerves that were particularly close to the vein and could not be separated from the vein by a surgical instrument except for the scalpel were enclosed together with the vein by a common fibrous sheath. In these cases, collagen fibres connected the perineurium with the venous adventitia. Therefore, it seems that in addition to technical failures that can potentially be avoided, a substantial percentage of nerve injuries during the treatment for varicose veins are associated with these microscopic anatomical features. Consequently, technical improvements cannot significantly reduce the rate of these complications, but instead a change of surgical strategy would be required. This may include precise preprocedural sonographic diagnostics, selective use of potentially dangerous ablative techniques, and a hybrid (a combination of techniques) approach to the treatment. However, such a modern management requires a good knowledge of anatomy of the nerves accompanying the veins, and knowledge of dangerous points and dangerous anatomical variants. Also, doctors should be familiar with different techniques of treatment for varicose veins. Importantly, non-thermal ablative methods, such as foam sclerotherapy, mechanico-chemical ablation (MOCA), or injection of cyanoacrylate glue, are very rarely associated with nerve injuries [19]. Similarly, nerves are rarely at risk during haemodynamic surgical techniques, such as CHIVA [37]. However, these methods are either less effective or more technically demanding, or more expensive in comparison with thermal ablative techniques and traditional surgery. Therefore, it is very unlikely that the above-mentioned safer methods will totally replace thermal ablation in the near future. It seems more likely that they will be primarily used in patients with smaller var- icosities or those presenting with contraindications for traditional surgery and thermal ablations. Consequently, an anatomical approach should be the main measure aimed at reducing the frequency of nerve injuries.

A tailored and sonography-guided approach to the treatment, selective use of thermal ablation, and hybrid procedures (e.g. thermal ablation of proximal part of the GSV and foam sclerotherapy of distal varicosities) should be preferred. This, however, would require good anatomical knowledge and training in the sonographic assessment of nerves. Unfortunately, at the moment both skills are not included in the vascular specialty curriculum. Besides, since currently many countries are facing a vascular specialist shortage, the treatments are often performed by non-specialists, which probably further contributes to the high rate of neurological adverse events after the treatment for varicose veins.

In order to lower the risk of nerve injuries, surgical excisions and thermal ablations should not be used for the treatment of incompetent segments of the GSV and SSV below the mid-calf. In this part of the lower leg these veins are typically very close to the saphenous or sural nerves, and in many patients - because of the microscopic structure - these nerves cannot be separated from adjacent veins, neither surgically nor by tumescent anaesthesia. Foam sclerotherapy and MOCA seem to be the only safe techniques in this part of the extremity. On the other hand, the proximal parts of the GSV and SSV in the majority of cases can be safely occluded using one of the thermal ablation techniques (laser, radiofrequency, or steam). However, thermal ablations should be accompanied by precise preprocedural sonographic mapping, including not only the veins but also adjacent nerves. A proximity to the saphenofemoral and saphenopopliteal junctions should be regarded as dangerous points. Thermal ablations should be performed in this region selectively and only after sonographic identification of local nerves, before and after administration of tumescent anaesthesia. In the case of unusual anatomy of the nerves, especially if they run very close to the target vein, MOCA or glue should be chosen instead of thermal ablation. Haemodynamic surgical procedures also seem to be a reasonable option in such patients.

\section{The authors declare no conflict of interest.}

\section{References}

1. Flu HC, Breslau PJ, Hamming JF, et al. A prospective study of incidence of saphenous nerve injury after total great saphenous vein stripping. Dermatol Surg 2008; 34: 1333-1339.

2. Jaworucka-Kaczorowska A, Oszkinis G, Huber J, et al. Saphenous vein stripping surgical technique and frequency of saphenous nerve injury. Phlebology 2015; 30: 210-216.

3. Markides GA, Subar D, Al-Khaffaf H. Litigation claims in vascular surgery in the United Kingdom's NHS. Eur J Vasc Endovasc Surg 2008; 36: 452-457. 
4. Subramonia S, Lees T. Sensory abnormalities and bruising after long saphenous vein stripping: impact on short-term quality of life. J Vasc Surg 2005; 42: 510-514.

5. Wood JJ, Chant H, Laugharne M, et al. A prospective study of cutaneous nerve injury following long saphenous vein surgery. Eur J Vasc Endovasc Surg 2005; 30: 654-658.

6. Beteli CB, Rossi FH, de Almeida BL, et al. Prospective, double-blind, randomized controlled trial comparing electrocoagulation and radiofrequency in the treatment of patients with great saphenous vein insufficiency and lower limb varicose veins. J Vasc Surg Venous Lymphat Disord 2018; 6: 212-219.

7. Doganci S, Yildirim V, Demirkilic U. Does puncture site affect the rate of nerve injuries following endovenous laser ablation of the small saphenous veins? Eur J Vasc Endovasc Surg 2011; 41: 400-405.

8. Park JY, Galimzahn A, Park HS, et al. Midterm results of radiofrequency ablation for incompetent small saphenous vein in terms of recanalization and sural neuritis. Dermatol Surg 2014; 40: 383-389.

9. Sanioglu S, Yerebakan H, Ozgen A, et al. Mid-calflevel as a puncture site is not safe enough for thermal ablation of the small saphenous vein. SAGE Open Med 2017; 5: 2050312117731474.

10. Spreafico G, Kabnick L, Berland TL, et al. Laser saphenous ablations in more than 1,000 limbs with long-term duplex examination follow-up. Ann Vasc Surg 2011; 25: 71-78.

11. Caggiati A, Bergan JJ, Gloviczki P, et al. Nomenclature of the veins of the lower limbs: an international interdisciplinary consensus statement. J Vasc Surg 2002; 36: 416-422.

12. Cavezzi A, Labropoulos N, Partsch H, et al. Duplex ultrasound investigation of the veins in chronic venous disease of the lower limbs--UIP consensus document. Part II. Anatomy. Eur J Vasc Endovasc Surg 2006; 31: 288-299.

13. Kerver AL, Leliveld MS, den Hartog D, et al. The surgical anatomy of the infrapatellar branch of the saphenous nerve in relation to incisions for anteromedial knee surgery. J Bone Joint Surg Am 2013; 95: 2119-2125.

14. Murakami G, Negishi N, Tanaka K, et al. Anatomical relationship between saphenous vein and cutaneous nerves. Okajimas Folia Anat Jpn 1994; 71: 21-34.

15. Beale RJ, Mavor AI, Gough MJ. Heat dissipation during endovenous laser treatment of varicose veins - is there a risk of nerve injury? Phlebology 2006; 21: 32-35.

16. Simka M, Pułtorak M. Varicose vein surgery performed in the tumescent local anesthesia (Klein's liposuction formula). Acta Phlebol 2004; 5: 57-59.

17. Akagi D, Arita H, Komiyama T, et al. Objective assessment of nerve injury after greater saphenous vein stripping. Eur J Vasc Endovasc Surg 2007; 33: 625-630.

18. Morrison C, Dalsing MC. Signs and symptoms of saphenous nerve injury after greater saphenous vein stripping: prevalence, severity, and relevance for modern practice. J Vasc Surg 2003; 38: 886-890.

19. Hirsch T. Varicose vein therapy and nerve lesions. Vasa 2017; 46: 96-100.

20. Sam RC, Silverman SH, Bradbury AW. Nerve injuries and varicose vein surgery. Eur J Vasc Endovasc Surg 2004; 27: 113-120.

21. Ozsoy MH, Tuccar E, Demiryurek D, et al. Minimally invasive plating of the distal tibia: do we really sacrifice saphenous vein and nerve? A cadaver study. J Orthop Trauma 2009; 23: 132-138.
22. Milleret R, Huot L, Nicolini P, et al. Great saphenous vein ablation with steam injection: results of a multicentre study. Eur J Vasc Endovasc Surg 2013; 45: 391-396.

23. Leliveld MS, Verhofstad MH. Injury to the infrapatellar branch of the saphenous nerve, a possible cause for anterior knee pain after tibial nailing? Injury 2012; 43: 779-783.

24. Kerver AL, van der Ham AC, Theeuwes HP, et al. The surgical anatomy of the small saphenous vein and adjacent nerves in relation to endovenous thermal ablation. J Vasc Surg 2012; 56: $181-188$.

25. Garagozlo C, Kadri O, Atalla M, et al. The anatomical relationship between the sural nerve and small saphenous vein: An ultrasound study of healthy participants. Clin Anat 2019; 32: 277-281.

26. Rodriguez-Acevedo O, Elstner K, Martinic K, et al. Hydrodisplacement of sural nerve for safety and efficacy of endovenous thermal ablation for small saphenous vein incompetence. Phlebology 2017; 32: 482-487.

27. Dangintawat $\mathrm{P}$, Huanmanop T, Agthong S, et al. Anatomy of the sural nerve related to calcaneal tendon, intermalleolar line and small saphenous vein. Int J Morphol 2016; 34: 380-384.

28. Uhl JF, Gillot C. Anatomy and embryology of the small saphenous vein: nerve relationships and implications for treatment. Phlebology 2013; 28: 4-15.

29. Rodriguez-Acevedo O, Elstner K, Zea A, et al. The sural nerve: sonographic anatomy, variability and relation to the small saphenous vein in the setting of endovenous thermal ablation. Phlebology 2017; 32: 49-54.

30. Atkin GK, Round T, Vattipally VR, et al. Common peroneal nerve injury as a complication of short saphenous vein surgery. Phlebology 2007; 22: 3-7.

31. de Alvarenga Yoshida R, Yoshida WB, Sardenberg T, et al. Fibular nerve injury after small saphenous vein surgery. Ann Vasc Surg 2012; 26: 729.e11-5.

32. Shahid KR, Dellon AL, Amrami KK, et al. Sciatic and peroneal nerve injuries after endovascular ablation of lower extremity varicosities: case reports and review of the literature. Ann Plast Surg 2015; 74: 64-68.

33. Schweighofer G, Mühlberger D, Brenner E. The anatomy of the small saphenous vein: fascial and neural relations, saphenofemoral junction, and valves. J Vasc Surg 2010; 51: 982-989.

34. Mendoza E. Ultrasound of the nerves and clinical implications. Phlebologie 2014; 43: 105-107.

35. Milone M, Di Minno MN, Maietta P, et al. Great saphenous vein stripping and nerve injury: the role of stripping direction. Int Angiol 2015; 34: 238-342.

36. Papakostas JC, Douitsis E, Sarmas I, et al. The impact of direction of great saphenous vein total stripping on saphenous nerve injury. Phlebology 2014; 29: 52-57.

37. Bellmunt-Montoya S, Escribano JM, Dilme J, et al. CHIVA method for the treatment of chronic venous insufficiency. Cochrane Database Syst Rev 2013; CD009648. 